\title{
Études juridiques comparatives et internationalisation du droit, 2002-2011
}

\section{Mireille Delmas-Marty}

\section{(2) OpenEdition}

1 Journals

Édition électronique

URL : https://journals.openedition.org/annuaire-cdf/1072

DOI : 10.4000/annuaire-cdf. 1072

ISBN : 978-2-7226-0325-7

ISSN : 2109-9227

Éditeur

Collège de France

Édition imprimée

Date de publication : 1 avril 2013

Pagination : 784-787

ISBN : 978-2-7226-0198-7

ISSN : 0069-5580

\section{Référence électronique}

Mireille Delmas-Marty, «Études juridiques comparatives et internationalisation du droit, 2002-2011 "

L'annuaire du Collège de France [En ligne], 112 | 2013, mis en ligne le 22 novembre 2013, consulté le 22 août 2022. URL : http://journals.openedition.org/annuaire-cdf/1072 ; DOI : https://doi.org/10.4000/ annuaire-cdf. 1072 
$\mathrm{M}^{\mathrm{me}}$ Mireille DeLMAS-MARTY, membre de l'Institut

(Académie des sciences morales et politiques)

\section{Études juridiques comparatives et internationalisation du droit, 2002-2011}

\section{RECHERCHE}

\section{L'internationalisation du droit : pathologie ou métamorphose de l'ordre juridique?}

Rencontre « inter-réseaux », 10-12 avril 2012, Collège de France, ministère des Affaires étrangères ${ }^{1}$.

Commencée dans le cadre du réseau franco-chinois sur l'élaboration de «principes directeurs internationaux de droit pénal », cette recherche a été élargie à d'autres pays (États-Unis et Brésil) et à l'ensemble du champ juridique sous le nom de « réseaux-ID ». Il ne s'agit plus d'élaborer des principes mais d'observer et de mettre en lumière, à partir de divers thèmes ${ }^{2}$, les processus d'internationalisation du droit (ID). À la fois interactifs et évolutifs, ces processus appelleraient de nouveaux modèles (ID se lit aussi imagination et droit).

Chacun des trois réseaux ayant tenu depuis 2005 plusieurs rencontres bilatérales (une douzaine au total), la rencontre de 2012 fut la première rencontre multilatérale.

Le caractère disparate des thèmes déjà étudiés ne permettait pas de passer directement à une synthèse. D'où le choix de reprendre ces thèmes à propos de trois catégories transversales - responsabilité, territorialité et souveraineté -, confiées à un rapporteur de chaque pays, puis discutées par l'ensemble des participants. Dans la mesure où ces catégories déterminent les contours de l'ordre juridique, elles permettent de préciser la question - pathologie ou métamorphose de l'ordre juridique ? - et d'esquisser une réponse: une métamorphose inachevée, mais suffisamment engagée pour en esquisser les contours.

La responsabilité internationale semble diluée par la diversification des acteurs (étatiques et non étatiques) exerçant un pouvoir global, mais la montée en puissance

1. Participants américains : Stephen Breyer, Diane Marie Amann, Vivian Curran, William Fletcher, Michel Rosenfeld; participants brésiliens : Luiz Olavo Baptista, Paulo Casella, Tarciso Dal Maso Jardim, Celso Lafer, Kathia Martin-Chenut, Francisco Rezek, Marília Sardenberg ; participants chinois : Jin Banggui, Li Bin, Li Qinglan, Lu Jianping, Zhang Li ; participants français: Robert Badinter, Paul Bouchet, William Bourdon, Guy Canivet, Emmanuel Decaux, Olivier Dutheillet de Lamothe, Antoine Garapon, Bruno Genevois, Régis de Gouttes, Gilbert Guillaume, Pierre Morel, Terry Olson, Alain Pellet, Hélène Ruiz-Fabri, Jean-Marc Sauvé.

2. Par exemple, la peine de mort, le droit de la concurrence, les technologies numériques, le changement climatique, la justice sociale, la justice pénale internationale, les juridictions militaires, le droit international des droits de l'homme, la criminalité économique et les atteintes à la dignité de la personne, le contrôle de la police, la gouvernance Internet, l'État et la propriété, l'État et la vie privée, la construction d'un État de droit. 
des juges (internationaux et nationaux) annonce peut-être une consolidation. Aussi la métamorphose de la responsabilité progresse, mais sous des formes et à des vitesses différentes selon qu'il s'agit des États ou des entreprises transnationales.

Quant au principe de territorialité, qui fonde l'ordre international, le débordement des frontières nationales paraît le remettre en cause, mais ce débordement pourrait aussi préparer une métamorphose vers un ordre juridique à géographie variable. Encore faut-il distinguer plusieurs situations selon que les frontières sont transgressées par l'extension de la compétence nationale (extraterritorialité); intégrées dans un ensemble global plus complexe, par superposition de compétences nationales et internationales (multi-territorialité); ou encore neutralisées par la dématérialisation de l'information qui appelle une coordination, et parfois une harmonisation, des compétences (trans-territorialité).

Reste la souveraineté, qui est sans doute la catégorie la plus sensible. Dès lors que l'État souverain reste l'acteur principal de l'ordre juridique, les interdépendances liées à la mondialisation sont des pathologies dans la mesure où elles affaiblissent le principe d'indépendance qui fonde l'idée même de souveraineté. À moins d'y voir l'amorce d'une métamorphose, de la souveraineté absolue en une souveraineté que l'on nomme parfois «partagée ». Le débat nous a toutefois conduits à préférer l'expression de souveraineté «solidaire», car elle implique moins l'exclusion des compétences traditionnelles que l'inclusion de nouvelles compétences, tendant à intégrer les solidarités liées à l'accroissement des interdépendances.

Pour qu'une telle métamorphose soit possible, il faut réussir à concilier interdépendances et indépendance, c'est-à-dire à organiser les réponses aux interdépendances tout en conservant le minimum d'indépendance sans lequel le concept même d'État serait menacé. D'où la nécessité de distinguer deux types d'interdépendances : d'une part les interdépendances imposées par les pratiques de la globalisation (qu'elles concernent les flux d'informations, les risques, par exemple sanitaires ou environnementaux, ou certains crimes globaux, comme le terrorisme ou les grands trafics) ; d'autre part, les interdépendances qu'on pourrait nommer «éthiques » car elles sont attachées au processus d'universalisation des droits de l'homme et relèvent de tout un ensemble de dispositifs internationaux que les États gardent la liberté d'intégrer : comparé à la situation européenne, le degré d'intégration reste faible dans les trois pays partenaires de notre recherche.

Nous sommes encore très loin d'une véritable souveraineté solidaire qui supposerait un accord sur les valeurs communes que chaque État aurait le devoir de protéger. Pourtant, les membres des trois réseaux, même les plus attachés au modèle souverainiste, ont été d'accord pour conclure (et parfois regretter) que le processus de métamorphose de la souveraineté était déjà amorcé. Combiné aux métamorphoses de la responsabilité et de la territorialité, il pourrait annoncer l'émergence d'un nouveau modèle d'ordre juridique (coresponsable, à géographie variable et solidaire) qui contribuerait à la gouvernance mondiale sans substituer aux États souverains la figure inquiétante d'un État mondial.

\section{PuBlications}

Delmas-Marty M., «L'émergence d'un ordre juridique mondial: pathologie ou métamorphose? », in Frydman B. et Meyer M. (dir.), De la nouvelle rhétorique à la logique juridique, Le centenaire de Charles Perelman, PUF, 2012, 203 sq. 
Delmas-Marty M., «Construire une citoyenneté à plusieurs niveaux », FauvarqueCosson B., Pataut E., Rochfeld J. (dir.), La citoyenneté européenne, SLC, 2012, 15 sq.

Delmas-Marty M., «L'ambivalence des nouvelles technologies », préface in Vergès E. (dir.), Droit, sciences et techniques, quelles responsabilités ?, Lexis Nexis, 2012, 3 sq.

Delmas-Marty M., «Conclusions », in Boy L., Racine J.B. et Sueur J.J. (dir.), Pluralisme juridique et effectivité du droit économique, Larcier, 2011, 500 sq.

Delmas-Marty M., «Sécurité et dangerosité », Revue française de droit administratif, 2011, 1096 sq.

Delmas-Marty M., «Propos conclusifs », Lavielle J.M., Bétaillen J., Prieur M. (dir.), Les catastrophes écologiques et le droit : échecs du droit, appels au droit, Bruylant, 2012, 573 sq.

Delmas-Marty M., «Préface », in Gaillard E., Générations futures et droit privé. Vers un droit des générations futures, LGDJ, 2012, XII sq.

Delmas-Marty M., «Préface », in Roman D. (dir.), La justiciabilité des droits sociaux : vecteurs et résistances, Dalloz, 2012.

\section{TRADUCTIONS}

- En anglais, La Chine et la démocratie (Fayard, 2007) : Delmas-Marty M. et Will P.E. (éd.), China, Democracy, and Law: A Historical and Contemporary Approach, Leiden, Brill, 2012 ; traduction de N. Norberg.

- En turc, Le crime contre l'humanité (PUF, 2009) : Insanliga Karsi Suc (éd. Iletisim Yayinlari, Istanbul, 2012) (trad. Berna Akkiyal).

\section{CONFÉRENCES ET ENTRETIENS}

- «Les forces imaginantes du droit », Moscou, Collège français, 4 octobre 2011.

- «Humaniser la mondialisation », Lisbonne, Institut universitaire, 11 octobre 2011.

- «Sociétés de la peur ou communauté de destin», Paris, Fondation des sciences politiques, 18 décembre 2011.

- «L'émergence d'un ordre juridique mondial ? », communication, Académie des sciences morales et politiques, 18 décembre 2011.

- «Par-delà le relatif et l'universel : le rôle du droit », Rabat, Centre Jacques Berque, 15 février 2012.

- «L'Humanisme juridique à l'épreuve de la mondialisation », Genève, La société de lecture, 13 mars 2012.

- «Internationalization of Law: Diversity, Perplexity, Complexity », Washington D.C., American Society of International Law, 106 ${ }^{\text {th }}$ Annual meeting, 30 mars 2012 ; publié dans American Journal of International Law, 2012.

- «Humanité, espèce humaine et droit pénal », Rome, 24 mai 2012, discours de réponse lors de la remise du prix Silvia Sandano.

- «Les Forces imaginantes du droit», débat avec Antoine Jeammaud et Isabelle Sayn, CERCRID, CNRS et Université de Saint-Etienne, 1 $1^{\text {er }}$ juin 2012.

- «L'internationalisation du droit», entretien avec Alain Supiot, IEA Nantes, 19 juin 2012, Esprit, 2012. 


\section{COLLOQUeS}

- «Ordering Legal Pluralism», key note speech in The European China Law Studies Association, Annual General Conference, Law and Justice: China's practices in a Global Context, Fondation des sciences politiques, Paris, 28-29 septembre 2011.

- «L'harmonisation des règles de droit », table ronde franco-chinoise, Fondation Victor Segalen, Paris, 29-30 septembre 2011.

- «Sécurité et dangerosité », Les pièges de la sécurité, Académie des sciences morales et politiques, 17 octobre 2011, (cf. Rev. française de droit administratif, 2011, précité).

- «Les forces imaginantes du droit», débat avec Peter Kemp, 30 e Symposium of Eco-ethica, Le nouvel ordre du monde, Paris, 24 octobre 2011 ; paru dans Eco-ethica, vol. 2, 2012.

- «Conclusions », Droit à la vérité et justice de transition dans les pays du cône sud, 1-2 décembre 2011, Faculté de droit de l'Université de Saô Paulo (Brésil).

- «L'humanisme juridique et la diplomatie culturelle », Institut français et Collège de France, Paris, 12-13 décembre 2011.

- «Formes, normes et dogmes », La vie des formes, Colloque de rentrée du Collège de France, 14 décembre 2011 (publié dans Changeux J.-P., La vie des formes, Odile Jacob, 2012) ; également La Science et les normes : entre éthique et droit, Quatrième Université européenne d'été, IHEST, 27 août 2012 (à paraître).

- «Le procureur européen et les règles pénales modèles », discours inaugural, Un projet pour le parquet européen ?, Luxembourg, 13-15 juin 2012, à paraître.

- «Humaniser la mondialisation : une utopie dynamique et réaliste », Cabé Ch. (dir.), Atlas des utopies, Le Monde, hors-série, 2012.

\section{DISTINCTIONS}

- Docteur honoris causa de l'Institut national des sciences pénales (INACIPE), Mexico, 2012.

- «Prominent Woman in International Law Award», American Society of International Law, Washington D.C., 2012.

- Prix International Silvia Sandano, Rome, 2012.

- «Promotion Mireille Delmas-Marty », École du barreau de Poitiers, 2011-2012.

\section{Michael EDWARDS}

\section{Étude de la création littéraire en langue anglaise, 2002-2008}

\section{Publications}

Edwards M., «John Constable regarde, distrait » (poème), Christian Gardair, Ciel( $s$ ), Libourne, LIS 33, 2011, p. 18-20.

Edwards M., «Poésie et vérité », XVII-XVIII, n 68, 2012, p. 173-185.

Edwards M., «Le jeu du latin dans la poésie anglaise », Sans le latin, éd. Suzzoni, Aupetit, Fayard, Mille et une nuits, 2012, p. 123-138. 